\title{
Closed and Open Metabolic Cycles: Transition Time*
}

\author{
Antonio Sillero, Víctor García-Herrero \\ Departamento de Bioquímica, Facultad de Medicina, Instituto de Investigaciones Biomédicas Alberto Sols, \\ UAM/CSIC, Madrid, Spain \\ Email: antonio.sillero@uam.es
}

Received 25 January 2016; accepted 26 February 2016; published 29 February 2016

Copyright (C) 2016 by authors and Scientific Research Publishing Inc.

This work is licensed under the Creative Commons Attribution International License (CC BY). http://creativecommons.org/licenses/by/4.0/

(c) (i) Open Access

\begin{abstract}
A metabolic cycle can be viewed as a central core and its branches. The central core is here firstly considered as a pre-closed metabolic cycle (CMC), with a unique first substrate, but with no input or output of other components. By contrast, the metabolic cycles in nature are open metabolic cycles (OMC) with output and input of external substrates (through "metabolic branches"), modulating continuously the enzyme activities and the total concentration of their substrates thorough complex regulatory phenomena. In this work, the transition from a Closed to an Open metabolic cycle has been simulated by a consecutive entry and exit of two components through the catalytic action of two enzymes. It is known that after any alteration of the initial conditions, the cycles need a time to reach new equilibrium. We have measured the changes of transition time (T.T.) values in 81 models of CMC differing in $\mathrm{Km}$ or Vmax values. In general, the T.T. tends to be shorter in cycles with preponderant lower $\mathrm{Km}$ and higher Vmax values. Further, Mathematica refinement for the estimation of transition time from the data previously calculated can be obtained with the use of the command Interpolating Function.
\end{abstract}

\section{Keywords}

Metabolic Cycles, Equilibration Times, Kinetic Constants, Differential Equations, Mathematica

\section{Introduction}

The open metabolic cycles (OMC) can be considered as systems, with a permanent entry and exit of substrates (metabolites); in spite of this dynamic state they tend to maintain, between physiological ranges, the concentration of their components. The OMC cycles can be studied with different and complementary approaches, among

*Dedicated to Dr. Maria Antonia Günther on her 80th birthday. 
others by measuring the level of their components and analyzing potential changes in their concentration in different metabolic or nutritional conditions. However, these are cumbersome procedures and sometimes difficult to be implemented [1]-[3]. The advances in computational techniques have allowed a more comprehensive understanding of open metabolic cycles [1] [4]-[7].

Part of the experimental work from our laboratory had been lately centered on the mechanism of action of enzymes ligases [8], and more particularly on the ubiquitin-activating enzyme. In this last case, experimental and theoretical aspects were carried out in parallel, with the help of the Mathematica Program [9]; the occurrence of 19 hypothetical intermediate enzyme forms (EFs) and 22 different reactions were then contemplated [9].

These previous studies directed us to apply the Mathematica Program to the analysis of several biochemical processes, such as:

a) Simulation of linear pathways exploring the effect of changing Vmax and/or Km values of one or more enzymes of the pathway; the reservoir model for enzyme kinetics previously developed in our laboratory was adapted to visualize the effect of the retro-inhibition of the first enzyme of the pathway by the final product; the time needed to achieve half of the total synthesis of the final product was also addressed [10];

b) Simulation of metabolic cycles, usually composed of a number of interconnected substrates and the same number of enzymes. Two different types of cycles can be theoretically considered, depending on whether i) substrates and enzymes form a separate entity in itself, with no entry or exit of material (a closed metabolic cycle, or $\mathrm{CMC}$ ) or ii) a continuous interchange of material (input and/or output) between the substrates of the inner core cycle and other related metabolites takes place (open metabolic cycles, or OMC). For reasons of simplicity we have firstly approached a peculiar type of closed metabolic cycle (or pre-CMC) which reaches equilibrium, starting from a unique initial substrate [9]-[13].

In this work, the changes in the substrate profiles of a closed metabolic cycle (CMC) promoted by the input and/or output of material are shown, and a new correlation between CMC and OMC is obtained. Although by definition the metabolic cycles tend permanently to equilibrium, the time needed to get that situation has been mathematically explored in theoretical situations in which the time needed to get the equilibrium was measured in cycles starting with only one of its substrates(a) at a fixed concentration (of $12 \mathrm{mM}$ or $1 \mathrm{mM}$ ).

\section{Materials and Methods}

\subsection{Nomenclature}

The close metabolic cycle (CMC) here considered contains 6 substrates and 6 enzymes, with Michaelis-Menten kinetics (Figure 1). The substrates of CMC are named as (a), (b), (c), (d), (e) and (f); for convenience, (a) and (f), were considered located in the first and last position. The enzymes are named with the letter E sub indexed with both, their position in the cycle and the name of the substrate (E1a, E2b, E3c, E4d, E5e, E6f); the actual equation velocities of the enzymes of the CMC are named from v1 to v6. In order to facilitate computer calculation and writing, the kinetic constants ( $V \max$ and $\mathrm{Km}$ ) of those enzymes are named simply as (Va to $V \mathrm{f})$ and $(K a$ to $K \mathrm{f}$ ), i.e. with the letters $V$ and $K$ sub indexed with the name of the corresponding substrate.

The closed metabolic cycle (CMC) is transformed into an open metabolic cycle (OMC) (Figure 1) by: i) the input of an external substrate (xc), at a fixed concentration(in this case ((xc), $3 \mathrm{mM}$ ) which is transformed into substrate (c) of the CMC by the enzyme E7xc with the following nomenclature and values: actual velocity (v7); $V \max (V x c=1)$ and $\mathrm{Km}(\mathrm{Kxc}=1)$; ii) the output of a substrate $(\mathrm{e})$ of the CMC which is transformed into an external substrate (ew) by the enzyme E8ew with the following nomenclature and values for its kinetic constants: actual velocity (v8); V8max $(V e w=1)$ and $\mathrm{Km}(\mathrm{Kew}=1)$.

\subsection{General Mathematica Treatment}

Part of this treatment is similar to that previously followed in other works from this laboratory [10]-[12].

The general procedure is outlined in Table 1.

Part $A$ of Table 1 contains the actual equation velocity of the 8 enzymes involved (Figure 1 ): the six enzymes of the CMC (v1 to v6) plus the enzyme (E7xc) catalyzing the input of (c) from an external fixed source (xc, $3 \mathrm{mM}$ ), and the enzyme (E8ew) catalyzing the output of (e) to an different external substrate (ew).

Part $B$ of Table 1 contains the Vmax and Km values of all the enzymes of the ensemble (Figure 1) Note as the $V$ max values of the CMC start with a $V a=1.02$ with increments of 0.08 units up to a value of $V \mathrm{f}=1.42$; all enzymes present a Km value = 1. The enzymes E7xc and E8ew, catalyzing the input and output of substrates (c) 
Table 1. Mathematica protocols.

A)

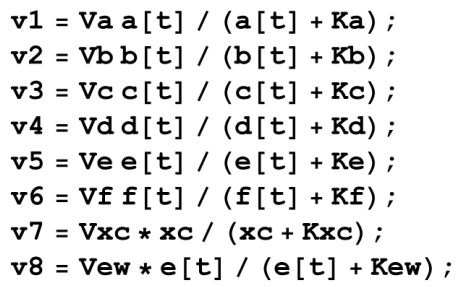

(E)

Plot [ Evaluate[a'[t] /.\%], Evaluate[b'[t] /.\%], Evaluate[c'[t] /.\%], Evaluate [d'[t] /.\%], Evaluate [e'[t] /.\%], Evaluate $[f(t t] / . \%]\},\{t, 0,500\}$, PlotRange $\rightarrow\{-1,1\}$, Plotstyle $\rightarrow$ \{Red, Gray, Thickness [0.01], Dashed, Gray, Gray $\}]$

and (e), respectively, have values of $V \max =\mathrm{Km}=1$. Finally a fixed and constant value of $3 \mathrm{mM}$ are assigned to the external substrate $(\mathrm{xc})$.

Part $C$ of Table 1 contains the instructions to calculate the substrate value concentration (profiles) along the reaction time. The first 3 lines of part $\mathrm{C}$ correspond to the commands needed to calculate substrate profiles (a to 


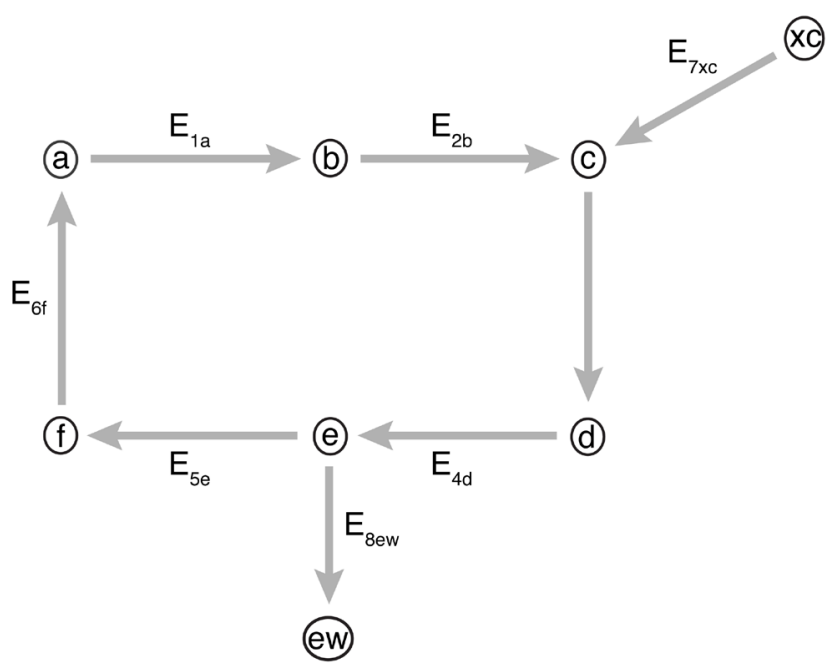

Figure 1. Jointly representation of a closed metabolic cycle (CMC) and an open metabolic cycle (OMC); theCMC is composed exclusively by the inter-conversion of 6 substrates (a) to (f) catalyzed by 6 enzymes E1a to E6f. A CMC can be converted into an OMC by the entry of (c) from (cx) and exitof (e), catalyzed by enzymes E7xc and E8e, respectively.

f) of the CMC, the time of calculation (Figure 2, Panel A) and the instructions to calculate the derivatives of those profiles (Figure 2, panel B).

The instructions for the Mathematica Program to draw Panels (C1, C2), (D1, D2) and (E1, E2) (Figure 3) and panels (F), (G) and (H) (Figure 4) are stated also in part C of Table 1. They convey substitution of (c' [t] = v2 $\mathrm{v} 3 \ldots$ or e' $[\mathrm{t}]=\mathrm{v} 4-\mathrm{v} 5 \ldots$ for the new values consigned below, in the same Table. The adequate moments for these substitutions will be indicted in the Text.

Part D contains the instructions to Plot the substrate profiles resulting in each case from the value assigned to the respective substrates.

Part E contains the instructions to Plot the derivatives of the profiles resulting in each case from the value assigned to the substrates (c') and (e').

\section{Results and Discussion}

\subsection{Profile of a Closed Metabolic Cycle}

1) A model of a closed metabolic cycle (CMC) composed of 6 substrates and 6 enzymes is firstly considered (Figure 1). It could be stresses that cycle was theoretically started with a unique input of substrate (a) $=12 \mathrm{mM}$ (Figure 2, panel A) an that at the very beginning the concentration of the rest of the substrates is zero (what could be considered as a pre-CMC); from 0 to 50 min the initial substrate (a) is distributed among the boxes of the cycle, reaching thereafter an equilibrium, according to the enzyme kinetic constants. These constants were selected as to give an articulate panorama of the substrate profiles. Here it is pertinent to recall that (in a cycle with $\mathrm{Km}=1$ for all the enzymes) the substrate concentrations, at equilibrium, are inversely related to the Vmax values of the matching enzymes; hence, substrate (a) reached the maximal concentration when the equilibrium is reached.

The derivatives of the substrate profiles are represented in panel B of Figure 2. The Mathematica instructions to draw this panel are in Table 1, part E.

The consequences of the input and output of materials to/from the cycle will be examined below in a cycle with the characteristics consigned above.

\subsection{Input into a Closed Metabolic Cycle (CMC), within Creasing Concentration of One of Its Substrates (c), from an External Source (xc) Catalyzed by an External Enzyme (E7xc)}

The input of one of the substrates (c) of CMC was carried out by an external enzyme (E7xc) catalyzing the 


\section{A}

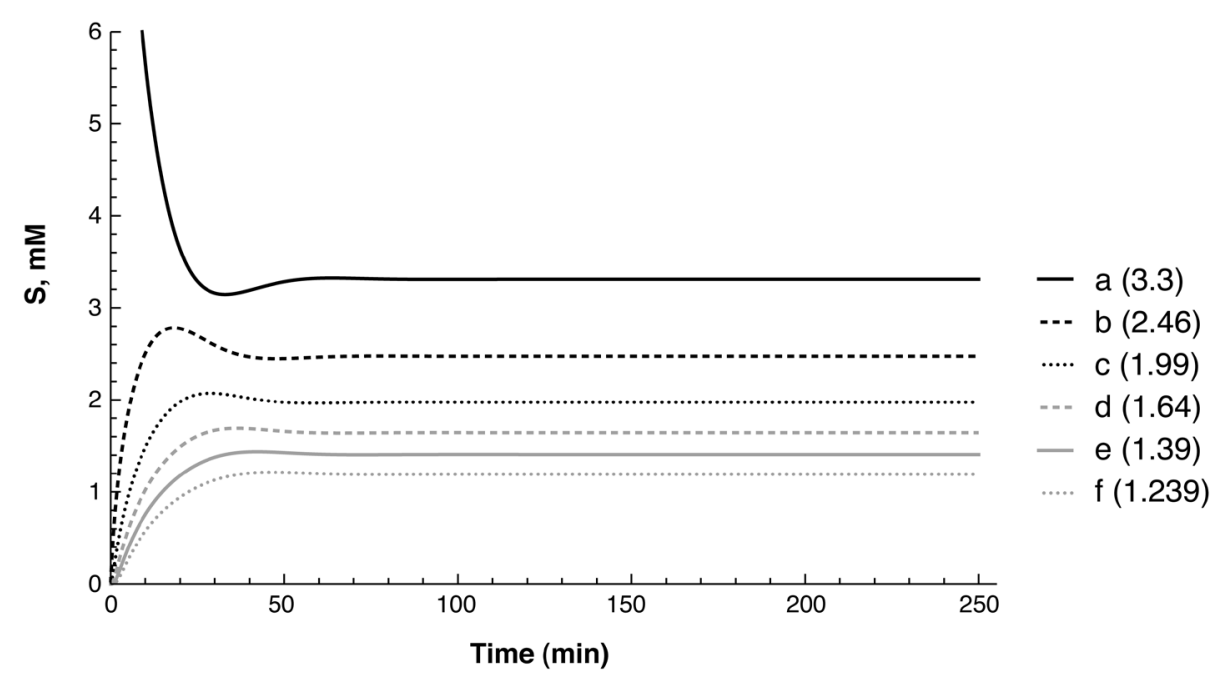

B

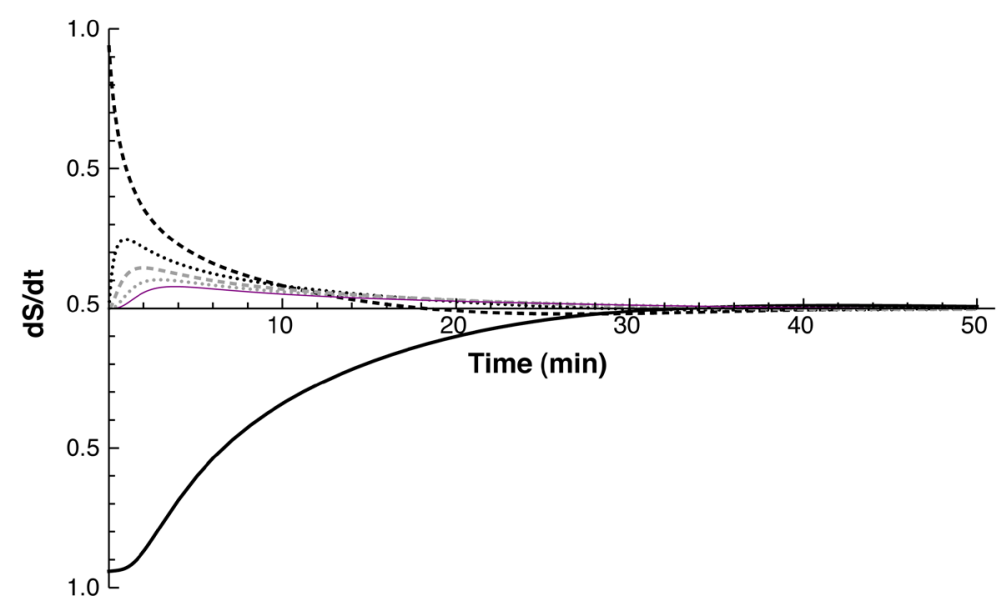

Figure 2. Example of a closed metabolic cycle (Figure 1) with the initial input of only one substrate (a, $12 \mathrm{mM}$ ). Profile of the 6 substrates(Panel A) and of their derivative functions (Panel B) The Mathematica protocol followed is in Table 1 . The $\mathrm{mM}$ concentration values reached by he substrates at equilibrium are indicated on Panel A.

transformation of (xc) into (c). The enzyme E7xc presented a Michaelis Menten kinetics with values of Vmax $(V \mathrm{xc}=1 \mathrm{micromoles} / \mathrm{min})$ and $\mathrm{Km}(\mathrm{Kxc}=1 \mathrm{mM})($ Table 1 , part A $)$.

In all cases (Figure 3), the simulation was started with the input of $12 \mathrm{mM}$ substrate (a). After the equilibrium of the CMC was reached (from 0 to $150 \mathrm{~min}$ ), the effect of the input of substrate (c) was examined during three periods of time, starting after the $150 \mathrm{~min}$ of reaction: $5 \mathrm{~min}$, (from t-150 to t-155) (Panel C1, C2); $10 \mathrm{~min}$ (from $\mathrm{t}-150$ to $\mathrm{t}-160$ (Panel D1, D2) or 30 min (from t-150 to t-180) (Panel E1, E2) (Figure 3). In these three cases, the changes were similarly approached, as shown in Table 1, lines: "Panel (C1, C2)- > c' [t]..."; "Panel (D1, D2)- > c'[t]..."; "Panel (E1, E2)- > c'[t]...". In these lines, the catalytic effect of enzyme E7xc during 5 min, 10 min or 30 min was represented. As an example, the instructions to draw panels (C1, C2) are: c' = v2 - v3 + v7 * Unit Step [t-150] - v7*Unit Step [t - 155]. For these operations, the value of (c' [t]) in the first line of part (C) must be replaced by the corresponding value indicated below in the same Table 1.

To better appreciate the results, the representation of the profiles of the substrates in Figure 3 are shown at two different scales in Panels (C1, D1 and E1) and in Panels (C2, D2 and E2).

Before the extra-input of substrate (c) by the external enzyme (E7xc), the concentration of the substrates at equilibrium (between 50 - $150 \mathrm{~min}$ ) is the same, panels C1, D1 and E1 (Figure 3), as visualized from the graphs, 
C1

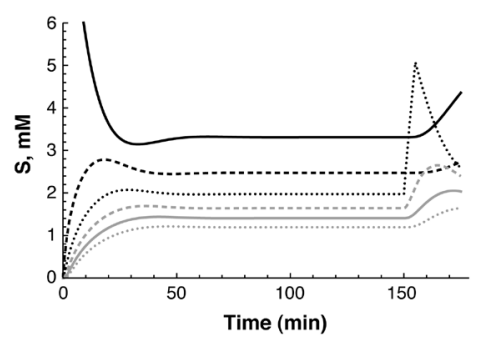

D1

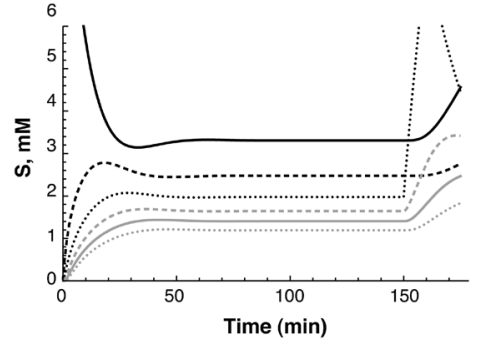

E1

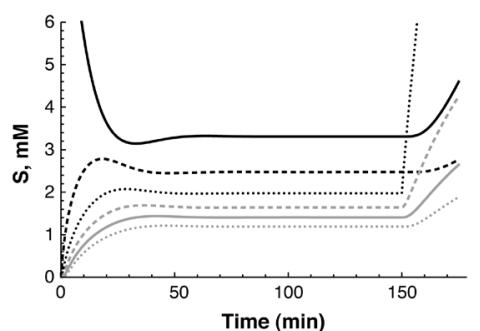

C2

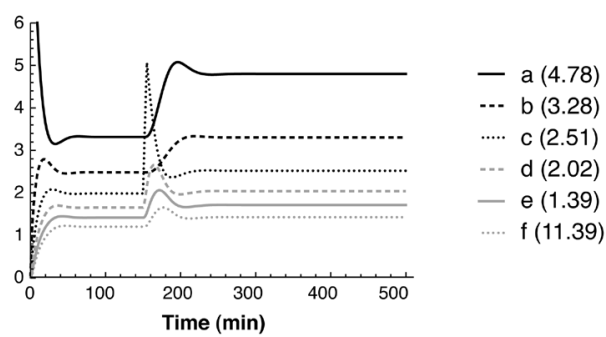

D2

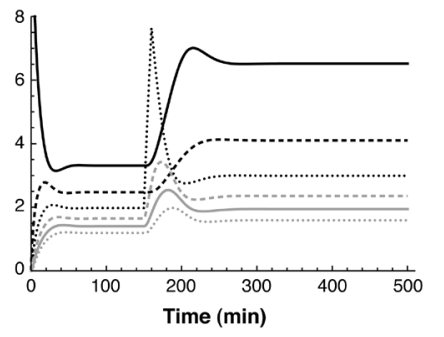

$$
\begin{aligned}
& \text { - a (6.54) } \\
& \text {... b (4.13) } \\
& \text { …. c (2.97) } \\
& \text {-.. d (2.37) } \\
& \text { - e(1.90) } \\
& f(1.58)
\end{aligned}
$$

\section{E2}

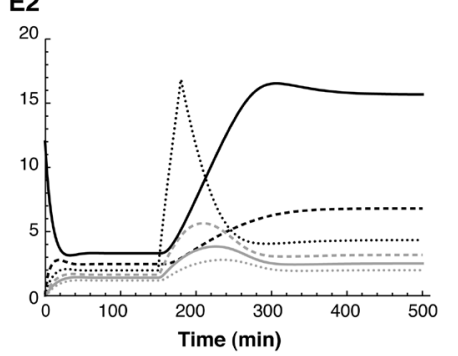

$$
\begin{aligned}
& \text { - a (15.69) } \\
& \text {-.- b (6.78) } \\
& \text {.... c (4.28) } \\
& \text {..- d (3.16) } \\
& \text { - e (2.51) } \\
& f(1.96)
\end{aligned}
$$

Figure 3. Substrate profiles of an open metabolic cycle OMC composed of 6 substrates and 6 enzymes (Figure 1) with he kinetic constant values depicted in Table 1 and with initial input of only one substrate (a, $12 \mathrm{mM}$ ). Once the equilibrium was reached (150 min) the input of substrate (c) was simulated from a fixed (invariant) concentration of (xc, $3 \mathrm{mM})$, catalyzed by an external enzyme (E7xc). The input of (c)was applied during 5 (Panel C), 10 (Panel D) and 30 min (Panel E). The instructions for the drawing of the substrate profiles of these panels are in Table 1 . To facilitate the interpretation of the results, the profile for the same substrates are represented at two differentscales,at the left $(\mathrm{C} 1, \mathrm{D} 1, \mathrm{E} 1)$ and right part (C2, D2, E2) of the figure.

and indicated here between brackets (a, 3.33), (b, 2.44), (c, 1.99), (d, 1.62), (e, 1.38) and (f, 1.19). Immediately after the input of (c) by enzyme (E7xc), the concentration of (c) reaches the maximal level, a value proportional to the duration of the input (5, 10 or $30 \mathrm{~min}$ ) (Figure 3, panels C2. D2 and E2). After a new transition time was attained, substrate (c) and the other substrates reached new levels, all of them inversely related to the maximal velocity of its proper enzyme in the cycle, as indicated at the right side of panels C2, D2, E2 (Figure 3).

\subsection{Effect of Both the Continuous Input of One Substrate (c), and the Output of a Different Substrate (e) from a Metabolic Cycle}

As in 3.2 the simulation was initiated with the input of $12 \mathrm{mM}$ substrate (a); after transition (at $150 \mathrm{~min}$ ) of the hypothetical CMC, the simulation of the OMC was firstly started by the input, at $150 \mathrm{~min}$ of reaction, of substrate (c) by enzyme E7xc during 5, 10 and 30 min (Panels F, G and H, Figure 4), and after the equilibrium was considered again complete, at 350 min of reaction, the cycle was subjected to the output of substrate (e) by enzyme E8ew, also during 5, 10 and 30 min (Figure 4). Both enzymes (E7xc, E8ew,) presented identical kinetic constants values $(\operatorname{Vmax}(\mathrm{Vxc}, \mathrm{Vew})=\mathrm{Km}(\mathrm{Kxc}, \mathrm{Kew})=1$, Table 1$)$. In order to better visualize these effects, panels F, G and H, are represented at different ordinate axes scales. The specific Mathematica commands to represent Panels F, G and H, are explicit in the lines of Table 1 named as: "Panel (F)- > c'[t]... and e'[t]...;" "Panel (G)- > c'[t]...; and e'[t]...;"; "Panel (H)- > c'[t]...; and e'[t]...;". 
$\mathbf{F}$

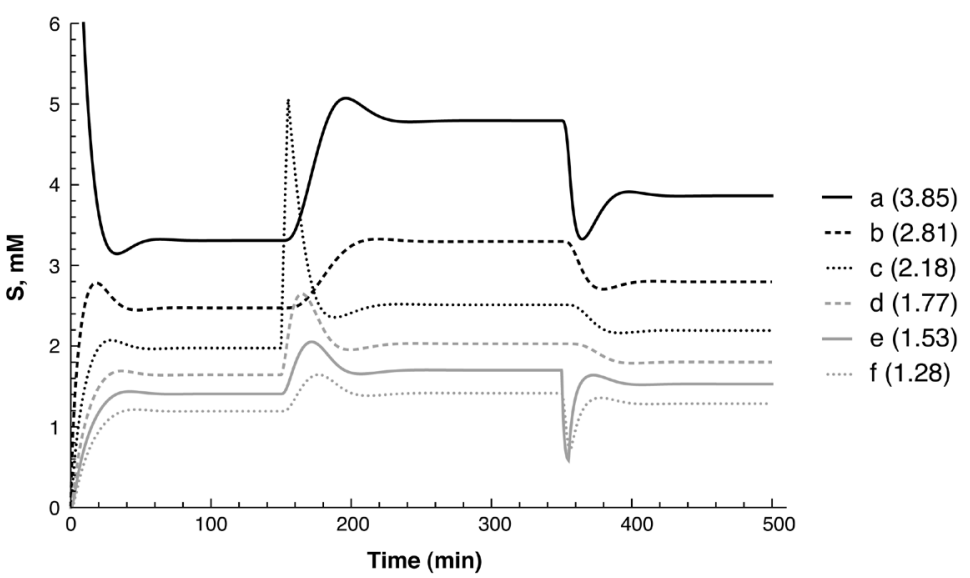

G

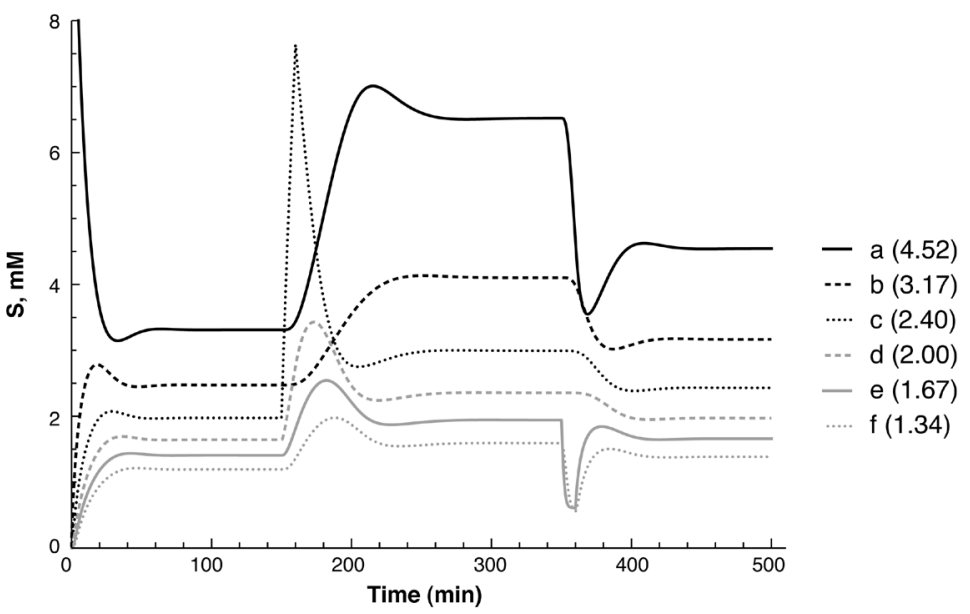

H

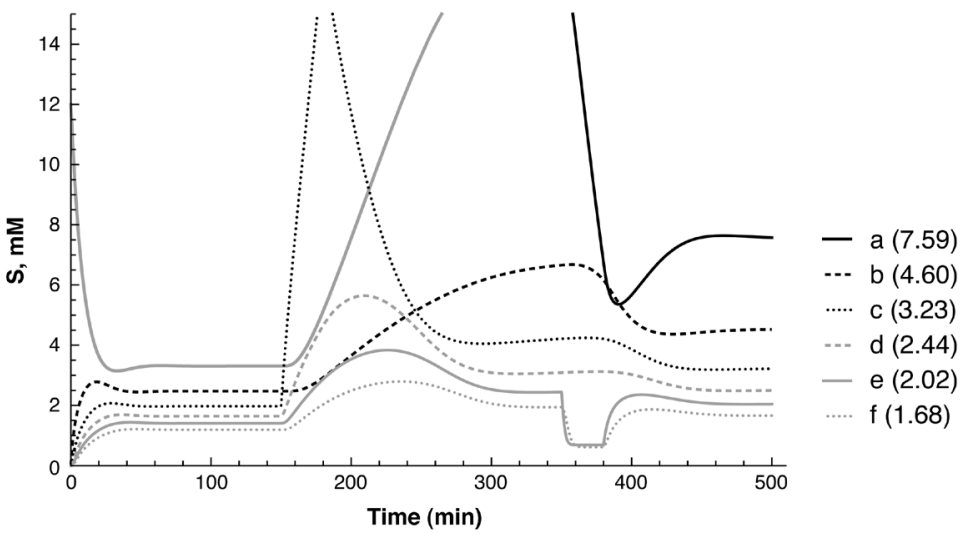

Figure 4. Substrate profiles of an open metabolic cycle OMC (Figure 1), starting with the input of a unique substrate (a, $12 \mathrm{mM}$ ). After 150 min of transition: i) the synthesis (input) of substrate (c), by an external enzyme (E7xc) catalyzing its synthesis from a fixed concentration of an external substrate (xc, $3 \mathrm{mM}$ ) for different time intervals (5, 10 and $30 \mathrm{~min}$, Panels F, G and H, respectively); ii) after a new equilibrium was established (around $300 \mathrm{~min}$ ), the output of the substrate (e) catalyzed by the an external enzyme (E8ex, Figure 1) was simulated. The duration of input and output of metabolites is the same in both cases: (Panel F, $5 \mathrm{~min}$ ), (Panel G, $10 \mathrm{~min}$ ), (Panel H, $30 \mathrm{~min}$ ). 
As expected, the initial reaction times (from 0 to $150 \mathrm{~min}$ ) in Panels C, D and E (Figure 3) and Panels F, G and $\mathrm{H}$ (Figure 4) are identical and correspond to the initial times of reaction. They are also identical following the input of substrate (c) during 5 min Panels (C and F), 10 min (Panels D and G) and during 30 min (Panels E and $\mathrm{H}$ ). The scenario of the profiles obtained is appreciably different after $350 \mathrm{~min}$ of reaction, and following the output of substrate (c) during 5 min (Panel F), 10 min (Panel G) or 30 min (H) (Figure 4).

As shown above (Figures 2-4), and elsewhere in a number of publications by others, the cycles tend to reach equilibrium to accommodate concentrations of internal and external concerned substrates, kinetic constants of the implicated enzymes, physiological conditions, etc. Be as it be, the potential changes in the composition of a cycle and its adaptation to new environments are not instantaneous and require a period of adjustment, or transition time (T.T.), defined here as the time required for all its substrates to reach a steady state situation.

The concentrations reached by each substrate at the end of the simulation is indicated at the right side of each panel.

\subsection{Transition Time (T.T.) of Metabolic Cycles Deduced from Graphical Representations}

From the results presented above and from those previously published [11] [12], changes in both the concentration of any substrate(s) or in the kinetic constants of any of the enzymes of CMC or OMC cycles bring about changes in the equilibrium among the substrates.

The possibilities of variations of the T.T. in different closed or open cycles is theoretically countless and depends, among others, on the number of enzymes, individual kinetic constant values, total amount of substrates concentration, initial concentrations, cellular locations, rates of input or output of substrates, etc. Nevertheless, the T.T. can be calculated following the protocols described in Table 1, and visualized for several cycle conditions in Figures 2-5.

The exploration of T.T. was simulated in 81 different types of CMC, similar to that presented in Figure 1; each cycle was composed by 6 substrates and 6 enzymes, with Michaelis Menten kinetics, and with the kinetic constant values shown in Table 2; in all cases, the cycles were started in the presence of a unique initial substrate ((a), $1 \mathrm{mM})$ and mathematically followed until the amount of the initial substrate was distributed at equilibrium among the previously empty boxes of the cycle. The values of the calculated T.T. values obtained in each case are shown in Table 2.

Table 2 contains two lines in black characters (horizontal and vertical) corresponding to Vmax and Km values, respectively. All the enzymes in each horizontal line (with standard characters) (Table 2) have the same Km value and all the enzymes in each vertical line have the same Vmax value. For example, the horizontal line corresponding to $\mathrm{Km}=0.1$ contains 9 T.T. values (from 42.08 to 1.08); these values are coming from 9 cycles, each one with 6 substrates and 6 enzymes; all the 6 enzymes of this row contain both the same $\mathrm{Km}$ value $(0.1 \mathrm{mM})$ and the $V \max$ value corresponding to the specified T.T. value.

As an example, the substrate profiles and their dS/dt values of the encircled T.T. $=8.97$ is represented in Figure 5 panels (panels I, J). The T.T. value in this case, and in the other cases can be grossly deduced from this type of graphical representations (Figure 5). Nevertheless we have developed two other methods for the numerical calculations of T.T. without any need of graphical representations (see below). The T.T. values consigned in Table 2 were obtained with following Protocols E and F (see below).

\subsection{Numerical Calculation of Transition Time (T.T.)}

\subsubsection{Protocol E}

a) It is evident that a cycle approaches equilibrium when the concentration of the metabolites tends to be constant (Panel I, Figure 5) or when the derivative function for all substrates, irrespectively of whether it is positive or negative, reaches insignificant values (between -0.005 and 0.005) (Panel J, Figure 5).

b) We took advantage of this fact representing the sum of the absolute values of the derivatives of the substrate profiles (Panel K, Figure 6); it may be worthy to recall that the absolute value of a number means to remove any negative sign in front of the number.

c) The command g [x_] (Figure 6) calculates, at successive times, (the sum of the absolute values of the derivatives of the substrate profiles) minus 0.005; the following command Find Root calculates the time when $\mathrm{g}(\mathrm{x})$ $=0$, which means that the sum of the absolute values of the derivatives is lower than 0.005 from that moment. The absolute values tend to smaller values with increasing reaction time, panel K (Figure 6). 


\section{I}

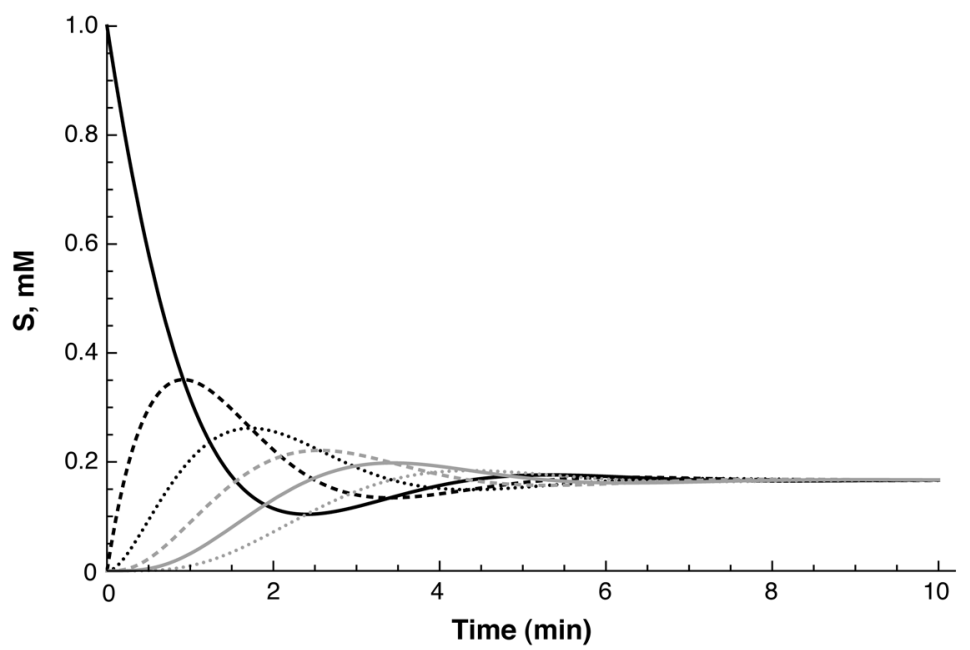

$\mathbf{J}$

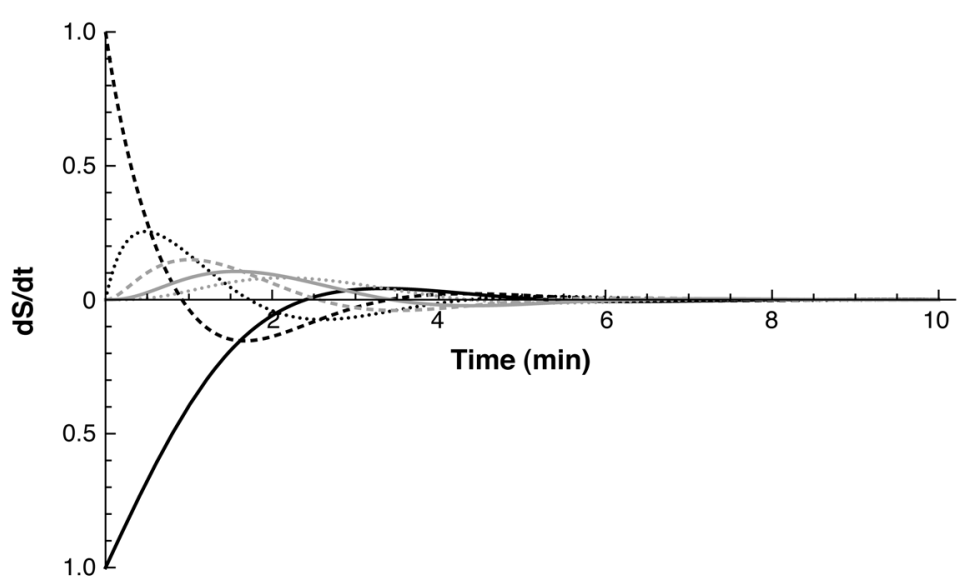

Figure 5. Graphical representation of the substrate profiles (Panel I) and their derivative functions (Panel J) of a CMC with a transition time of 8.97 (Table 2). As stated in the text, the transition time (T.T.) values shown in Table 2 were obtained in a CMC cycle composed of 6 substrates (a to f) and starting with a unique substrate concentration of (a), $1 \mathrm{mM}$. This particular cycle has the same $\mathrm{Km}$ value of 2, andVmax value of 3 for the six enzymes of the CMC cycle.

d) The final statement of this protocol ( $\{\mathrm{t}->8.977453671572034\}$ ' $)$ contains, in black characters, the calculated time value for this precise T.T.

\subsubsection{Protocol F}

Differs from Protocol E in the use of the square of the derivatives of the substrate profiles, instead of their absolute numbers (Panel $\mathrm{L}$ ). The aim in that case was to transform the negative values of the derivatives into positive values. The sum of the squares of the derivative values also tend to be smaller in the course of the reaction; the T.T. value calculated with this method is indicated in black characters at the bottom of this protocol Figure 6. The T.T. values obtained with Protocols E and F apparently differ in this particular case (8.97 and 7.79).

The horizontal lines parallel to the $\mathrm{x}$-axis, in panels $\mathrm{K}$ and L (Figure 6), were drawn to indicate two different views of T.T.: mathematical and biological.

The computer calculated value for T.T. corresponds to the exact point of intersection of the line representing the modified derivative with the $\mathrm{x}$-axis, as indicated by the two vertical arrows; the biological meaning could reflect, in this context, the tendency of the cycles to reach an equilibrium after, and during, an indefinite period of time. 


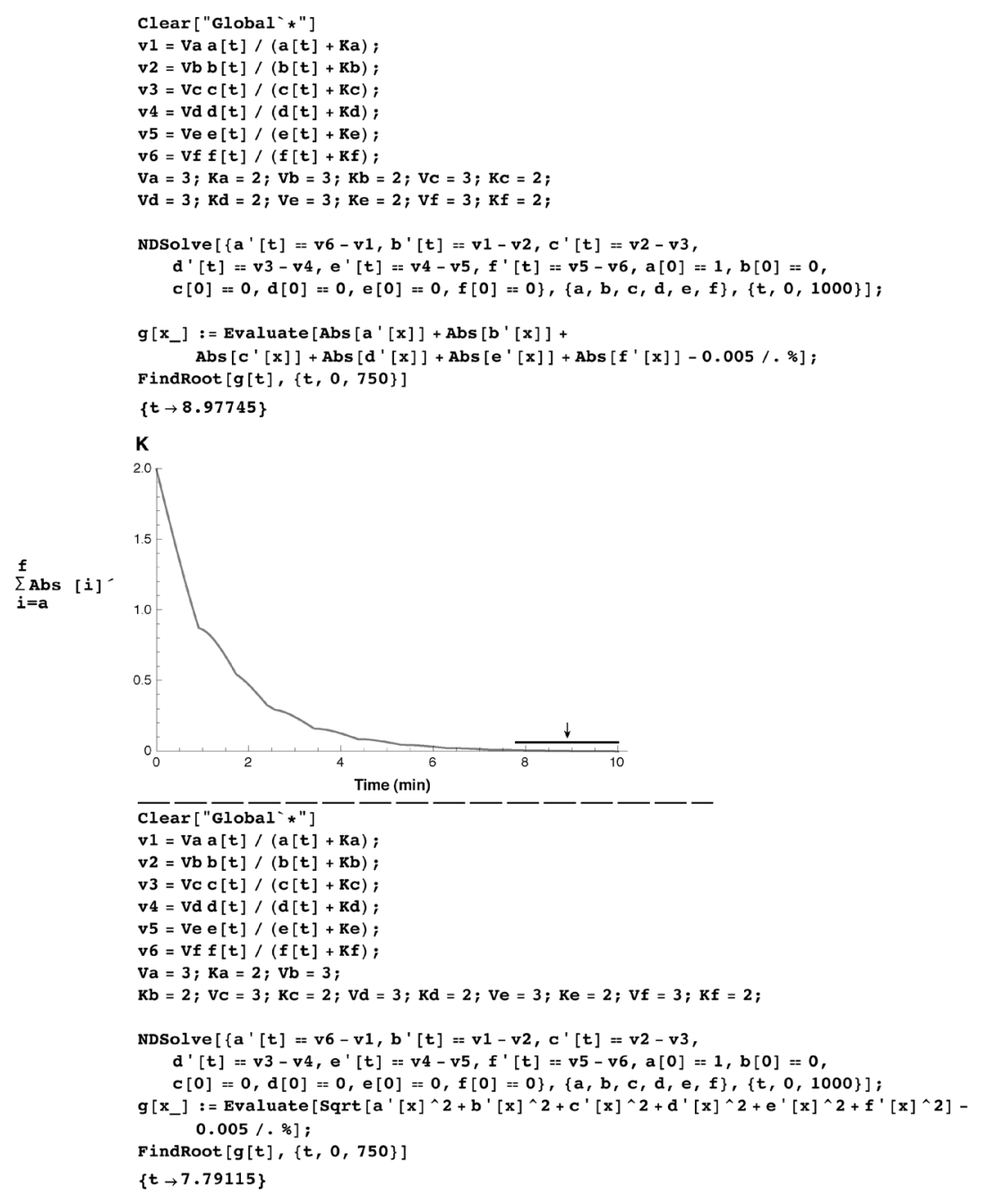

(a)

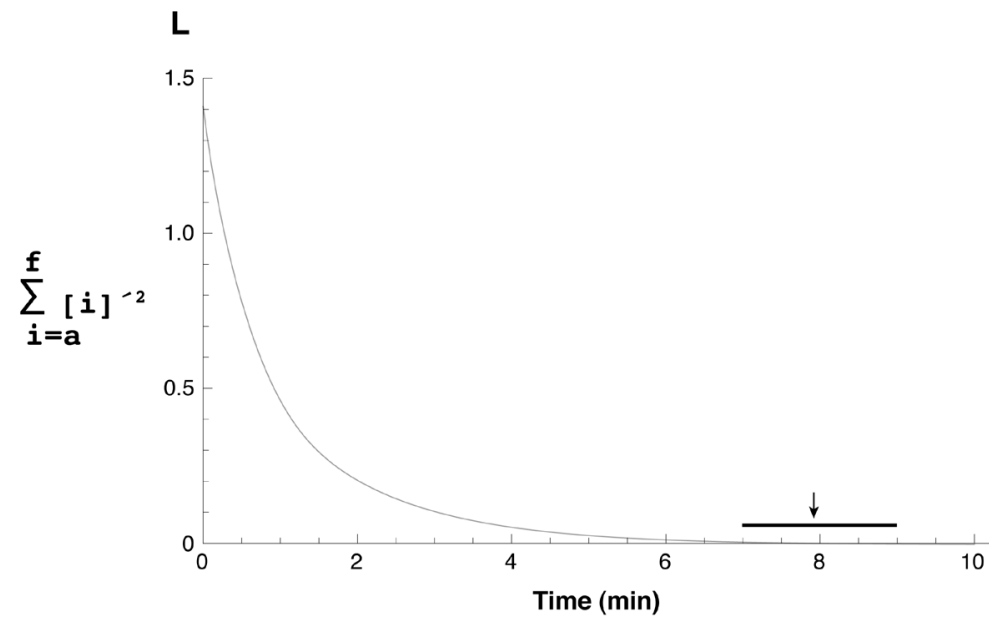

(b)

Figure 6. Mathematical protocols used to calculate numerically the transition times (T.T.) shown in Table 2. Protocols E and F were elaborated as described in the Text, in Table 1 and in the proper Figure 6. Panel K was drawn using the absolute values of the derivative functions; Panel L was elaborated using the sum of the square of the derivatives. 


\subsection{Influence of the Enzyme Kinetic Constants Values ( $V$ max and $\mathrm{Km}$ ) of a Closed Metabolic Cycle (CMC) on Its Transition Time (T.T.)}

An overview of the data of Table 2 is represented in Figure 7; the upper panel contains a three-dimensional representation of the T.T. of 45 cycles with Km values from 0.1 to 0.5 and Vmax values from 0.1 to 10 (see Table 2); the lower panel contains the T.T. representation of all the 81 cycles of Table 2. In both parts, three-dimensional scales were used with $\mathrm{Km}, V \max$ and transition times values in axes $\mathrm{x}, \mathrm{y}, \mathrm{z}$, respectively, Transition Time
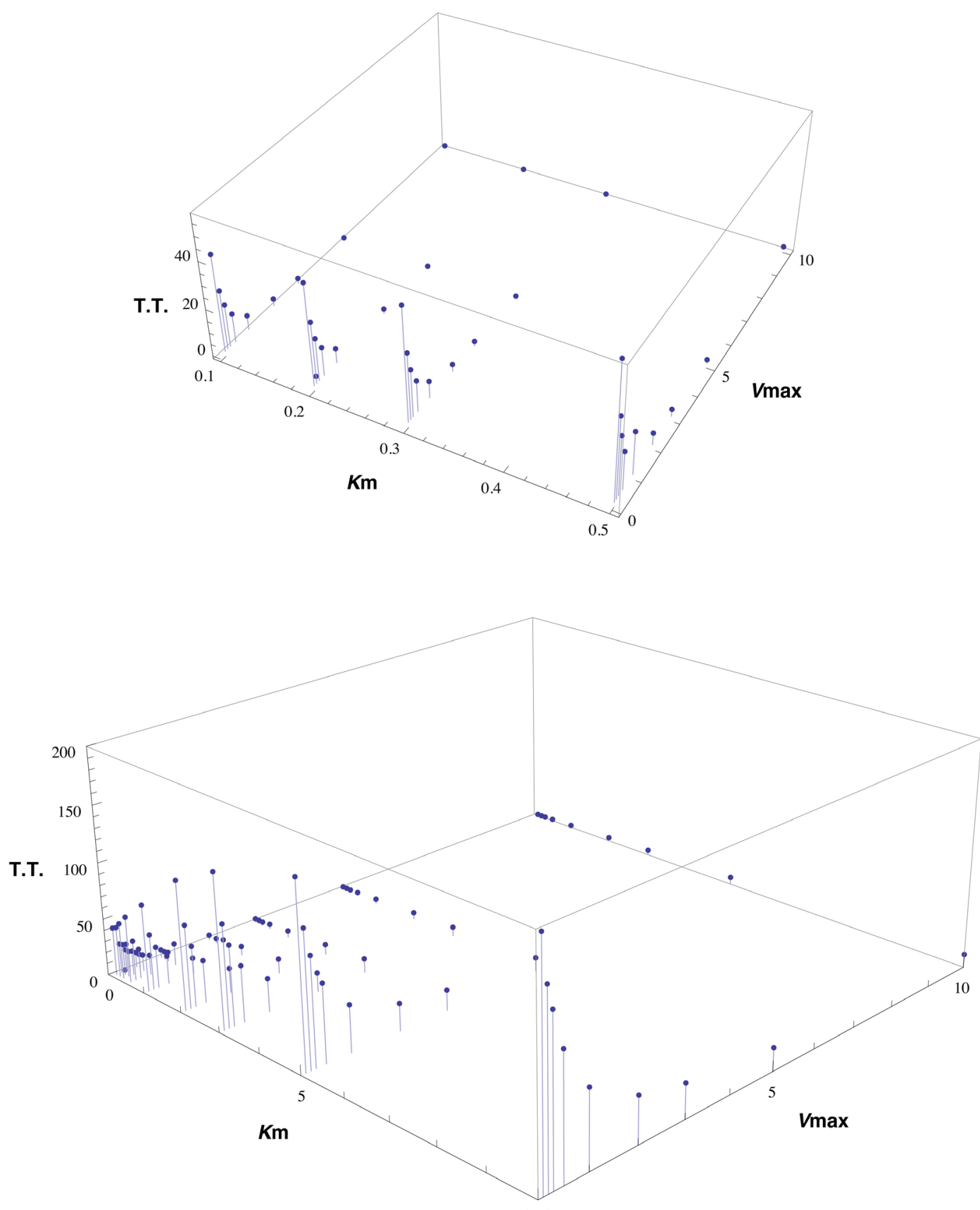

100

Figure 7. Three-dimensional representations of transition time (T. T.) versus enzyme kinetic constants (Km and $V \max$ ). The $\mathrm{Km}, V \max$ and transition times values are represented in axes $\mathrm{x}, \mathrm{y}, \mathrm{z}$, respectively. In the upper panel are represented 45 cycles with Km values from 0.1 to 0.5 and Vmax values from 0.1 to 10 (see Table 2); the lower panel contains the T.T. representations for all the 81 cycles of Table 2. 
Table 2. Transition time (T.T.) calculated for 81 different closed metabolic cycles (CMC), considering an unique initial substrate concentration of (a, I mM); the predetermined Vmax and Km values are shown in black characters in horizontal and vertical lines, respectively. Calculation of the T.T. is indicated in the Text.

\begin{tabular}{cccccccccccc}
\hline & & \multicolumn{7}{c}{ Vmax values } & & & \\
\hline & & $\mathbf{0 . 1}$ & $\mathbf{0 . 2}$ & $\mathbf{0 . 3}$ & $\mathbf{0 . 5}$ & $\mathbf{1}$ & $\mathbf{2}$ & $\mathbf{3}$ & $\mathbf{5}$ & $\mathbf{1 0}$ \\
\hline & $\mathbf{0 . 1}$ & 42.08 & 25.68 & 19.43 & 13.19 & 7.54 & 4.24 & 3.05 & 1.97 & 1.08 \\
& $\mathbf{0 . 2}$ & 44.52 & 26.82 & 19.07 & 12.87 & 7.52 & 4.24 & 2.95 & 1.92 & 1.04 \\
& $\mathbf{0 . 3}$ & 48.49 & 29.10 & 21.53 & 14.33 & 8.21 & 4.60 & 3.24 & 2.08 & 1.14 \\
Kalues & $\mathbf{1}$ & 76.48 & 48.62 & 35.90 & 24.50 & 14.05 & 7.91 & 5.72 & 3.72 & 2.04 \\
& $\mathbf{2}$ & 112.92 & 72.94 & 52.92 & 36.95 & 21.54 & 12.64 & $\mathbf{8 . 9 7}$ & 5.90 & 3.25 \\
& $\mathbf{3}$ & 135.53 & 89.84 & 70.35 & 49.18 & 29.16 & 16.82 & 12.19 & 7.95 & 4.46 \\
& $\mathbf{5}$ & 160.54 & 118.24 & 94.16 & 68.08 & 41.12 & 24.07 & 17.74 & 11.77 & 6.61 \\
& $\mathbf{1 0}$ & 196.87 & 157.81 & 137.83 & 105.01 & 66.29 & 39.96 & 29.88 & 20.09 & 11.46 \\
\hline
\end{tabular}

values are remarked as straight columns in both panels of Figure 7. It is clear that, in these conditions, the transition time tends to be relatively shorter with increasing Vmax and decreasing Km values and, vice versa, relatively larger with decreasing Vmax or increasing Km values, as deduced from a simple inspection of Table 2 and Figure 7.

\section{Mathematica Refinement for the Estimation of Transition Time (E. T.) Obtained by Extrapolation of Data Previously Calculated}

The data presented in Table 2 contains series of T.T. values, calculated one by one, of metabolic cycles, composed of 6 substrates and 6 enzymes, with known Km, and Vmax values. Based on these data, it is possible to deduce more T.T. values by application of the command Interpolating Function. With this method, Mathematica produces an implicit polynomial formula, which approximates, with reasonable accuracy, the requested T.T. values, with the condition that they lie in the range of $\mathrm{Km}$, and Vmax parameters given in Table 2. To illustrate this point, we have used the $4 \times 4$ (T.T. data) comprised between both Km, and Vmax values of 0.1 to 0.5 to produce the corresponding polynomial. With this new method, two new sets of T.T. values were obtained (Table 3). In one of the set, the $V \max$ was maintained between the prefixed 0.1 to 0.5 parameters and the $\mathrm{Km}$ values oscillated from 0.15 to 0.45 with increments of 0.1 units. In the other set, $\mathrm{Km}$ was maintained between the prefixed 0.1 to 0.5 and Vmax oscillated from 0.15 to 0.45 . The correlation among $V \max , \mathrm{Km}$ and T.T. values obtained with this new procedure agrees quite well with the results presented in Table 2 and Figure 7; i.e. lower T.T. figures were obtained with decreasing $\mathrm{Km}$ and increasing Vmax values. The advantage of this method is that it allows estimation with great precision of a large amount of T.T. values starting from small pieces of data within a shorter period of time.

\section{Concluding Remarks}

This work was started considering a closed metabolic cycle (Figure 1) composed of six substrates (a to f) and six enzymes (E1a to E6f). Although closed cycles do not exist in metabolism because they would be useless, i.e. in a permanent state of equilibrium, the one presented here, named pre-close metabolic cycle, is a theoretical model with special useful characteristics: i) at time zero only one substrate is present (a, see examples at $12 \mathrm{mM}$, or $1 \mathrm{mM}$ ), which is later distributed among the previously considered empty boxes of the cycle according to the kinetic properties of its constituent enzymes; ii) the time needed to get the new situation has been name as "transition time" or the time needed for the cycle to get an equilibrium (see 3.1 and Figure 2); the pre-closed metabolic cyclic allowed a very scrupulous way to calculate the dependence of the transition time with the kinetic constants of the constituent enzymes as explained in the text (see 3.4, 3.5, Table 2, Figure 6 and Figure 7); iii) once stabilized, the model was easily transformed into a true open metabolic cycle simulating the input of an increasing concentration of only one substrate (c) from an external source, during three periods of time (see 3.2 
Table 3. Further Mathematica refinement for the estimation of Transition Time (T.T.) obtained by extrapolation of data previously calculated in Table 2 The $T$. T. values of the first Table were obtained using real Vmax values (Table 2) and imaginary Km values. The T.T. values of the second Table were obtained using real $\mathrm{Km}$ values (Table 2) and imaginary Vmax Values.

\begin{tabular}{|c|c|c|c|c|}
\hline \multirow[t]{2}{*}{$\mathrm{Km}$} & \multicolumn{4}{|c|}{ Vmax } \\
\hline & 0.1 & 0.2 & 0.3 & 0.5 \\
\hline 0.15 & 43.06 & 26.07 & 18.75 & 12.72 \\
\hline 0.25 & 46.36 & 27.85 & 20.09 & 13.46 \\
\hline 0.35 & 50.81 & 30.50 & 23.11 & 14.29 \\
\hline 0.45 & 55.62 & 33.49 & 25.53 & 16.83 \\
\hline \multirow[t]{2}{*}{$\mathrm{Km}$} & \multicolumn{4}{|c|}{$V \max$} \\
\hline & 0.15 & 0.25 & 0.35 & 0.45 \\
\hline 0.1 & 32.23 & 21.66 & 18.22 & 15.86 \\
\hline 0.2 & 34.10 & 22.02 & 17.32 & 14.86 \\
\hline 0.3 & 36.89 & 24.27 & 20.02 & 17.28 \\
\hline 0.5 & 44.22 & 29.16 & 17.29 & 20.55 \\
\hline
\end{tabular}

and Figure 3) or by the consecutive input and output of two of its components (see 3.3 and Figure 4); note as in Figure 3 and Figure 4 the final concentration of the substrates of the cycle was calculated following the methods described in Table 1, where are condensed the Mathematica procedures used along this work.

The importance to scale time to visualize several biological aspects have been addressed in several texts [14][18].

The time of reaction in coupled enzyme systems has been examined in multiple publications. Two main aspects have been treated in those works: the time needed in a chain of coupled enzyme reactions to get a measurable steady state production of product [19]-[21] and the implications of the transition time required for an enzyme system to reach a new state after a perturbation [22].

In our view, the studies on transition time presented here are scarce in the bibliography, probably because it is very difficult or even impossible to measure experimentally these parameters in cycles, which are continuously evolving with the entry and exit of metabolites.

\section{Acknowledgements}

We thank Dr. María Antonia Günther Sillero for helpful comments on the manuscript, and Javier Pérez and Silvia Cuena for very helpful drawing assistance. This investigation was supported by Grants BFU2009-08977 and SAF 2915-64864-R.

\section{References}

[1] Alberty, R.A. (2011) Enzyme Kinetics, Rapid-Equilibrium Applications of Mathematica. Hoboken. http://dx.doi.org/10.1002/9780470940020

[2] Morris Jr., S.M. (2002) Regulation of Enzymes of the Urea Cycle and Arginine Metabolism. Annual Review of Nutrition, 22, 87-105. http://dx.doi.org/10.1146/annurev.nutr.22.110801.140547

[3] Albe, K.R. and Wright, B.E. (1992) Systems Analysis of the Tricarboxylic Acid Cycle in Dictyostelium discoideum. II. Control Analysis. The Journal of Biological Chemistry, 267, 3106-3114.

[4] Korla, K. and Mitra, C.K. (2014) Modelling the Krebs Cycle and Oxidative Phosphorylation. Journal of Biomolecular Structure and Dynamics, 32, 242-246. http://dx.doi.org/10.1080/07391102.2012.762723

[5] Chalhoub, E., Hanson, R.W. and Belovich, J.M. (2007) A Computer Model of Gluconeogenesis and Lipid Metabolism in the Perfused Liver. American Journal of Physiology: Endocrinology and Metabolism, 293, 1676-1686.

[6] Maher, A.D., Kuchel, P.W., Ortega, F., de Atauri, P., Centelles, J. and Cascante, M. (2003) Mathematical Modelling of 
the Urea Cycle. A Numerical Investigation into Substrate Channelling. European Journal of Biochemistry/FEBS, 270, 3953-3961.

[7] Bachmann, C. and Colombo, J.P. (1981) Computer Simulation of the Urea Cycle: Trials for an Appropriate Model. Enzyme, 26, 259-264.

[8] Günther Sillero, M.A., de Diego, A., Perez-Zuniga, F.J. and Sillero, A. (2008) Synthesis of Bisphosphonate Derivatives of ATP by T4 DNA Ligase, Ubiquitin Activating Enzyme (E1) and Other Ligases. Biochemical Pharmacology, 75, 1959-1965.

[9] López-Cánovas, F.J., Cánovas, F., Günther Sillero, M.A. and Sillero, A. (2010) Mathematical Model for the Ubiquitin Activating Enzyme E1. Journal of Biomedical Science and Engineering, 3, 274-286.

[10] López-Cánovas, F.J., Gomes, P.J. and Sillero, A. (2013) Mathematica Program: Its Use to Simulate Metabolic Irreversible Pathways and Inhibition of the First Enzyme of a Metabolic Pathway as Visuaized with the Reservoir Model. Computers in Biology and Medicine, 42, 853-864.

[11] García-Herrero, V., López-Cánovas, F.J. and Sillero, A. (2014) A Model Metabolic Cycle Simulated with the Mathematica Program. Journal of Biomedical Science and Engineering, 7, 286-295. http://dx.doi.org/10.4236/jbise.2014.75031

[12] García-Herrero, V. and Sillero, A. (2015) Pedagogical View of Model Metabolic Cycles. Biochemistry and Molecular Biology Education, 43, 468-475. http://dx.doi.org/10.1002/bmb.20920

[13] Sillero, A. and García-Herrero, V. (2015) Theoretical Evaluation of Both Unknown Substrate Concentrations and Enzyme Kinetic Constants of Metaboloc Cycles. Journal of Biomedical Science and Engineering, 8, 479-489. http://dx.doi.org/10.4236/jbise.2015.88045

[14] Fell, D. (1997) Understanding the Control of Metabolism. Portland Press; Distributed by Ashgate Pub. Co. in North America, London; Miami Brookfild, VT.

[15] Cornish-Bowden, A. (2001) Fundamental of Enzyme Kinetics. Portland Press Ltd., London.

[16] Klipp, R., Herwig, R., Kowald, A., Wierling, C. and Lehrach, H. (2005) System Biology in Practice. Wiley-VCH Verlag GmbH \& Co, Winheim (FRG). http://dx.doi.org/10.1002/3527603603

[17] Alon, U. (2006) An Introduction to System Biology. Design Principles of Biological Circuits. CRC Press, Taylor \& Francis Group, London.

[18] Gunawardena, J. (2014) Time-Scale Separation-Michaelis and Menten’s Old Idea, Still Bearing Fruit. The FEBS Journal, 281, 473-488. http://dx.doi.org/10.1111/febs.12532

[19] Storer, A.C. and Cornish-Bowden, A. (1974) The Kinetics of Coupled Enzyme Reactions. Applications to the Assay of Glucokinase, with Glucose 6-Phosphate Dehydrogenase as Coupling Enzyme. The Biochemical Journal, 141, $205-209$. http://dx.doi.org/10.1042/bj1410205

[20] Keleti, T. and Welch, G.R. (1984) The Evolution of Enzyme Kinetic Power. The Biochemical Journal, 223, $299-303$. http://dx.doi.org/10.1042/bj2230299

[21] Easterby, J.S. (1981) A Generalized Theory of the Transition Time for Sequential Enzyme Reactions. The Biochemical Journal, 199, 155-161. http://dx.doi.org/10.1042/bj1990155

[22] Llorens, M., Nuno, J.C., Rodriguez, Y., Melendez-Hevia, E. and Montero, F. (1999) Generalization of the Theory of Transition Times in Metabolic Pathways: A Geometrical Approach. Biophysical Journal, 77, 23-36. http://dx.doi.org/10.1016/S0006-3495(99)76869-4 